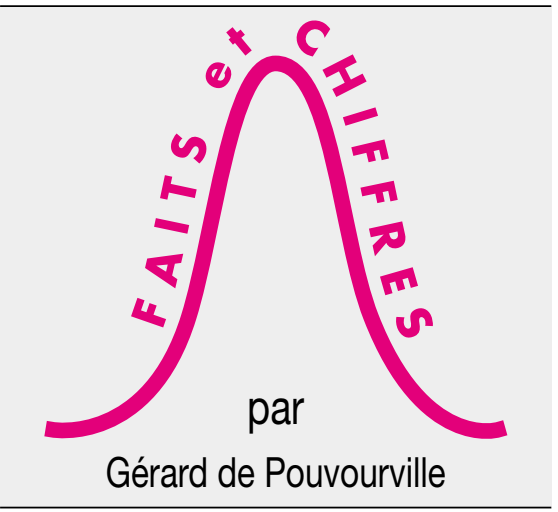

médecine/sciences 1998 ; 14 : 640-1

\title{
La reproduction sociale des inégalités de santé
}

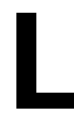
'existence, et la persistance d'inégalités devant la maladie et la mort liées à la position dans la hiérarchie sociale sont des faits reconnus par de nombreuses recherches en santé publique, et cela depuis plusieurs années. Il n'en reste pas moins que les causes de ces inégalités restent obscures. S'agit-il de l'adage "mieux vaut être riche et bien portant que pauvre et malade ", qui suggère que ce sont surtout les conditions matérielles de bien-être qui vont déterminer non seulement l'état de santé tout au long de la vie, mais aussi la durée de celle-ci ? Cette thèse a au moins le mérite de la simplicité, et d'une apparente vérité quand on pense aux conditions de travail aux extrêmes de l'échelle sociale. Qui oserait nier que l'ouvrier du bâtiment, exposé pendant toute sa vie professionnelle à un travail dur et intense physiquement, à la manipulation d'outils invalidants dans un environnement sale, dangereux et bruyant, payé de surcroît sur des contrats instables, ne risque pas une usure prématurée par rapport à un cadre ?

Pourtant, ce type d'explication est loin d'épuiser les observations faites sur les inégalités de santé, en liaison avec diverses mesures d'inégalités sociales. Par exemple, l'étude sur les fonctionnaires anglais publiée par Marmot et al. en 1991 [1] portait sur des individus qui n'étaient pas dans une situation matérielle difficile : ces fonctionnaires disposaient tous d'une stabilité de l'emploi et de revenus qui les mettaient à l'abri de

de bureau ne les exposait pas à des risques majeurs. Or, Marmot et al. [1] ont mis en évidence de façon très robuste un gradient tant en matière de mortalité que de morbidité en fonction de la position occupée par chacun dans la hiérarchie. Certes, ces différentiels face à la maladie et la mort pourraient être le résultat d'un processus de sélection naturelle : ceux qui atteignent le haut de la pyramide sont aussi ceux dont le patrimoine initial en santé et le comportement leur a garanti une meilleure santé que les autres, condition d'une meilleure réussite sociale. Hélas, il semble bien que ce patrimoine initial, et les comportements qui vont avec, sont largement déterminés par le milieu familial : si effet de sélection naturelle il y a, il est sérieusement renforcé par un effet de reproduction sociale.

\section{Une cohorte suivie 33 ans}

A l'appui de cette thèse, Chris Power et Sharon Matthews [2] ont publié en novembre 1997 dans le Lancet un article dont les résultats s'appuient sur l'analyse de la cohorte des personnes nées en Angleterre, Écosse et au Pays de Galles en 1958. Ces personnes ont été suivies jusqu'à l'âge de 33 ans, avec des observations recueillies à $7,11,16$, et 23 ans. Ont été recueillies des données relatives à la présence de certaines affections, à l'état de santé perçu. Mais l'originalité de la cohorte réside dans la collecte de données relatives à la position sociale des parents de ces enfants, repérés en quatre classes reflétant la nature de l'emploi, et à l'existence de facteurs de risques liés à la vie de la famille : tabagisme des parents, et de la mère pendant la grossesse, habitudes diététiques, consommation d'alcool, conditions générales d'habitat, niveau de revenu, conflits parentaux, etc. Les quatre classes sont les suivantes: les classes I et II recouvrent des emplois dits de professionnels, la classe III regroupe les emplois qualifiés «manuels» et «non manuels», et les classes IV et V les travailleurs peu ou pas qualifiés. Il s'agit donc, dans ce travail, de mettre en relation l'environnement familial dans un sens très général et l'état de santé à 33 ans des personnes de la cohorte.

L'intérêt du travail de recherche réside bien évidemment dans le suivi longitudinal sur des longues périodes d'une cohorte, mais également dans l'étude de caractéristiques sociales, économiques, de conditions de vie matérielles, de sécurité psychologique offertes par les familles des personnes suivies au cours de leurs premières années. Ainsi, la thèse de la «sélection des meilleurs " est éliminée par la prise en compte de l' " héritage familial». Ce que constate l'article, c'est le parallélisme d'un gradient des états de santé par classe sociale, et des gradients de plusieurs facteurs de risques pour la santé pour ces mêmes classes sociales. Par exemple, l'état de santé perçu, l'existence de problèmes respiratoires, la présence d'une maladie de longue durée invalidante, la détresse psychologique sont d'autant plus fréquents que l'on descend 
l'échelle sociale (Tableau I, où ont été rapportés les différences significatives à $5 \%$ sur une sélection de problèmes de santé).

Or, on retrouve un gradient social de même sens lorsque l'on observe le poids à la naissance : pour les personnes issues de familles d'ouvriers ou d'employés peu ou pas qualifiés, le poids et la taille des enfants à la naissance est plus faible. Mais la prévalence d'obésité à l'âge adulte s'inverse. Les conditions générales d'habitat et d'environnement matériel sont évidemment bien meilleures pour les classes des professionnels ou périprofessionnels : mieux vaut être riche que pauvre. Ainsi, à l'âge de 7 ans, environ $18 \%$ des répondants des classes I et II vivent dans un logement trop petit, contre $60 \%$ des répondants des classes IV et V. L'alimentation du bébé au sein est moins fréquente au bas de l'échelle sociale : $24 \%$ des répondants des classes I et II n'ont pas été nourris au sein, contre environ $38 \%$ pour les répondants des classes IV et V. Mais le tabagisme de la mère pendant la grossesse, le tabagisme des parents au cours de l'adolescence et le tabagisme des personnes suivies elles-mêmes suivent les mêmes gradients, avec un écart de $10 \%$ entre les classes extrêmes. Cela est vrai également des habitudes alimentaires. Le climat familial - les conflits entre parents et la force du réseau de soutien social, le soutien scolaire offert aux enfants aux divers âges - est également très lié à la classe sociale à la naissance. Enfin, ce gradient social explique également bien l'insertion sociale et professionnelle à l'âge adulte. A 33 ans, environ $4 \%$ des répondants dont les parents appartenaient aux classes I et II reçoivent des indemnités de chômage, contre $10 \%$ des répondants des classes $\mathrm{IV}$ et $\mathrm{V}$.

\section{Quelles conclusions peut-on en tirer?}

Il s'agit certes dans l'article de Power et Matthews [2] de la mise en parallèle de gradients de facteurs de risques en fonction de la classe sociale à la naissance, et de gradients d'états de santé. Mettre en relation n'est cependant pas démontrer. C'est pourquoi les auteurs s'appliquent dans la discussion à montrer la plausibilité de la relation entre certains facteurs de risque, comme le tabagisme de la mère durant la grossesse et le tabagisme «familial»

\section{Tableau I}

MORBIDITÉ À 33 ANS EN FONCTION DE LA CLASSE SOCIALE À LA NAISSANCE : PRÉVALENCE EN \%

\begin{tabular}{|c|c|c|c|c|c|}
\hline \multicolumn{6}{|l|}{ Hommes } \\
\hline & $\underset{\text { Classe }}{\text { Cl }}$ & $\underset{\text { II }}{\text { Classe }}$ & $\begin{array}{c}\text { Classe } \\
\text { III }\end{array}$ & $\begin{array}{c}\text { Classes } \\
\text { IV-V }\end{array}$ & $\begin{array}{c}\% \\
\text { Total }\end{array}$ \\
\hline Santé perçue mauvaise & 8,5 & 10,7 & 13,6 & 17,2 & 13,1 \\
\hline Maladie longue invalidante & 5,1 & 5,9 & 6,2 & 7,6 & 6,2 \\
\hline Détresse psychologique & 4,2 & 5,1 & 7,4 & 7,7 & 6,6 \\
\hline Symptômes respiratoires & 16 & 17,1 & 21,4 & 24,7 & 20,6 \\
\hline \multicolumn{6}{|l|}{ Femmes } \\
\hline & $\begin{array}{c}\text { Classe } \\
\text { I }\end{array}$ & $\begin{array}{l}\text { Classe } \\
\text { II NM }\end{array}$ & $\begin{array}{c}\text { Classe } \\
\text { III M }\end{array}$ & $\begin{array}{c}\text { Classes } \\
\text { IV-V }\end{array}$ & $\begin{array}{c}\% \\
\text { Total }\end{array}$ \\
\hline Santé perçue mauvaise & 7,7 & 9,3 & 15,1 & 18,8 & 13,9 \\
\hline Maladie longue & 12,4 & 13 & 13,8 & 17,3 & 14,2 \\
\hline Maladie longue invalidante & 4,7 & 5,7 & 5,7 & 8,6 & 6,1 \\
\hline Détresse psychologique & 6,3 & 11,5 & 12,5 & 15,4 & 11,9 \\
\hline Symptômes respiratoires & 12,4 & 10,4 & 17 & 22 & 16,6 \\
\hline
\end{tabular}

et la fréquence plus importante de problèmes respiratoires à l'âge adulte. Si l'on suit les auteurs, tout se passerait comme si la position sociale à la naissance allait entraîner une accumulation d'expositions à des facteurs de risque de nature diverse au cours de l'enfance, entraînant un état de santé plus dégradé à l'âge adulte, et aussi une moins bonne capacité d'entreprendre une ascension sociale: les inégalités se reproduisent d'une génération à l'autre. Mais ce n'est pas seulement parce que les personnes les plus défavorisées à la naissance sont en moins bonne santé, c'est aussi parce que durant leur enfance ils ont été soumis à un environnement affectif de moins bonne qualité que leurs alter ego des classes les plus favorisées.

Les auteurs sont moins convaincants quand ils essaient de tirer les conséquences de cette analyse en termes de politiques de santé. Ils insistent avant tout sur la nécessité d'une prise en charge renforcée de la petite enfance d'un point de vue affectif, et d'un soutien scolaire. Pourtant, l'article suggère que la correction elle-même des inégalités pourrait être un remède efficace, ou tout du moins, la compensation relative de certains des handicaps liés à cette inégalité : l'insécurité des revenus, les conditions d'habitat, la qualité du réseau de soutien social. En tout état de cause, l'article suggère au moins que l'accroissement de ces inégalités promet à la génération suivante une dégradation de son état de santé. Ce résultat pourrait être médité en France : il est bien possible que la génération qui a suivi celle du baby-boom - les enfants des baby-boomers -, qui ont vécu une période d'accroissement des inégalités et d'insécurité, présentent à l'âge adulte plus de problèmes de santé que leurs parents

\section{RÉFÉRENCES}

1. Marmot MG, Smith GD, Stansfeld S, Patel C, North F, Head J, White I, Brunner E, Feeney A. Health inequalities among British civil servants : the Whitehall II study. Lancet 1991 ; 337 : 1387-93.

2. Power C, Matthews S. Origins of health inequalities in a national population sample. Lancet 1997 ; 350 : 1584-9. 